Journal of Data Science 723-742 , DOI: 10.6339/JDS.201810_16(4).00004

\title{
THE INVERSE WEIBULL GENERATOR OF DISTRIBUTIONS: PROPERTIES AND APPLICATIONS
}

\author{
Amal S. Hassan*, Said G. Nassr² \\ *Mathematical Statistics, Institute of Statistical Studies and Research, Cairo University, Egypt \\ ${ }^{2}$ Faculty of Business Administration and International Marketing, Sinai University, Egypt
}

\begin{abstract}
In this paper, we introduce a new family of univariate distributions with two extra positive parameters generated from inverse Weibull random variable called the inverse Weibull generated (IW-G) family. The new family provides a lot of new models as well as contains two new families as special cases. We explore four special models for the new family. Some mathematical properties of the new family including quantile function, ordinary and incomplete moments, probability weighted moments, Rènyi entropy and order statistics are derived. The estimation of the model parameters is performed via maximum likelihood method. Applications show that the new family of distributions can provide a better fit than several existing lifetime models.
\end{abstract}

Keywords: Inverse Weibull distribution; Moments; quantiles; Inverse Weibull-G family.

\footnotetext{
${ }^{*}$ Corresponding author. Amal S. Hassan

Email: dr.amalelmoslamy@gmail.com
} 


\section{Introduction}

The inverse Weibull (IW) distribution is an important probability distribution which can be used to analyze the life time data with some monotone failure rates. It is suitable model to describe degradation phenomena of mechanical components as mentioned by Keller and Kamath (1982). According to Nelson (1982), the IW distribution provides a good fit to several data sets such as the times to breakdown of an insulating fluid subject to the action of a constant tension. Many works have been made about the IW distribution; for example, Calabria and Pulcini $(1990,1994)$ dealt with parameter estimation of the distribution. Some useful measures for the inverse Weibull distribution have been discussed by Jiang et al. (2001). Mahmoud et al. (2003) derived order statistics from inverse Weibull distribution. Based on lower record values, Sultan (2008) derived the Bayesian estimators and obtained the estimators of the reliability and hazard functions for the unknown parameters of the inverse Weibull distribution. Based on Type-II censored data, Kundu and Howlader (2010) studied the Bayes estimates of the unknown parameters of IW distribution under a squared error loss function. Hassan and Al-Thobety (2012) provided optimum simple failure step stress partially accelerated life tests for the model parameters and acceleration factor for inverse Weibull model. Hassan et al. (2015) discussed the constant-stress partially accelerated life test for inverse Weibull model based on multiple censored data.

The random variable $X$ has an inverse Weibull distribution if its cumulative distribution function (cdf) takes the form

$$
F(t)=e^{-\theta^{\beta} t^{-\beta}} ; t, \theta, \beta>0,
$$

where, $\theta$ and $\beta$ are the scale and shape parameters respectively. The corresponding probability density function (pdf) is given by

$$
f(t)=\beta \theta^{\beta} t^{-\beta-1} e^{-\theta^{\beta} t^{-\beta}} ; t, \theta, \beta>0
$$

In recent years, some endeavors have been made to define new generators of continuous distributions from classic ones to provide great flexibility in modelling data in several applied areas. Several generated families have been suggested by several authors; see for examples, beta-G by Eugene et al. (2002), gamma-G by Zografos and Balakrishanan (2009), Kumaraswamy-G by Cordeiro and de Castro (2011), generalized beta-G by Alexander et al. (2012), exponentiated generalized (exp-G) by Cordeiro et al. (2013), transformedtransformer (T-X) by Alzaatreh et al. (2013), Weibull-G by Bourguignon et al. (2014), Kumaraswamy Weibull-G by Hassan and Elgarhy (2016 a), exponentiated Weibull-G by Hassan and Elgarhy (2016 b), additive Weibull-G by Hassan and Hemeda (2016), Type II half logistic by Hassan et al. (2017 a), generalized additive Weibull-G by Hassan et al. (2017 b), and power Lindley-G by Hassan and Nassr (2018) among others.

Alzaatreh et al. (2013), defined the T-X family of distributions as follows

$$
F(x)=\int_{0}^{W[G(x)]} r(t) d t
$$


where, $r(t)$ is the pdf of a random variables $T$ and $W[G(x)]$ be a function of the cdf of any random variables $X$.

In this article, we provide a new family of distributions using inverse Weibull as a generator with the hope that it will attract a wider application in some areas. This paper can be organized as follows. In Section 2, we provide a formation of the IW-G family. Four special models of IW-G family are defined in Section 3. Some useful expansions for the pdf and cdf of IW-G family are derived in Section 4. In the same section, explicit expressions for the moments, probability weighted moments, order statistics and quantile function are obtained. Estimation of the model parameters using maximum likelihood method is performed in Section 5. Section 6 provides two applications to two real data sets are presented to illustrate the potentially of the new family. Section 7 ends with some concluding remarks.

\section{Inverse Weibull-G Family}

In this section, we display the formation of the IW-G family of probability distributions. The cdf, reliability function, hazard rate function and cumulative hazard rate are derived.

Based on T-X generator, the distribution function of IW-G family is derived by replacing the generator, $r(t)$, in (3) by inverse Weibull generator defined in (1), with $W[G(x)]=G(x ; \xi) / \bar{G}(x ; \xi)$ as the following

$$
F(x)=\int_{0}^{\frac{G(x ; \xi)}{\bar{G}(x ; \xi)}} \beta \theta^{\beta} t^{-\beta-1} e^{-\theta^{\beta} t^{-\beta}} d t=e^{-\theta^{\beta}\left[\frac{G(x ; \xi)}{\bar{G}(x ; \xi)}\right]^{-\beta}}
$$

where, $\bar{G}(x ; \xi)=1-G(x ; \xi)$. The associated pdf of the IW-G family can be written as follows

$$
f(x)=\frac{\beta \theta^{\beta} g(x ; \xi)[G(x ; \xi)]^{-\beta-1}}{[\bar{G}(x ; \xi)]^{-\beta+1}} e^{-\theta^{\beta}\left[\frac{G(x ; \xi)}{\bar{G}(x ; \xi)}\right]^{-\beta}} ; x, \theta, \beta>0
$$

A random variable $X$ having IW-G density function (5) is denoted by $X \sim \mathrm{IW}-\mathrm{G}$.

For $\beta=1 \mathrm{IW}-\mathrm{G}$ reduces to new generated family called the inverse exponential-G family, for $\beta=2$ the IW-G reduces to another generated family named the inverse Rayleigh-G family. Also, for $\theta=1$, the IW-G reduces to the odd Frèchet-G family presented by Haq and Elgarhy (2018).

Furthermore, the reliability function; $\bar{F}(x)$, hazard rate function; $h(x)$ and cumulative hazard rate function $H(x)$ are, respectively, given by

$$
\bar{F}(x)=1-e^{-\theta^{\beta}\left[\frac{G(x ; \xi)}{\bar{G}(x ; \xi)}\right]^{-\beta}},
$$




$$
\begin{gathered}
h(x)=\frac{\beta \theta^{\beta} g(x ; \xi)[G(x ; \xi)]^{-\beta-1}}{[\bar{G}(x ; \xi)]^{-\beta+1}} e^{-\theta^{\beta}\left[\frac{G(x ; \xi)}{\bar{G}(x ; \xi)}\right]^{-\beta}}\left[1-e^{-\theta^{\beta}\left[\frac{G(x ; \xi)}{\bar{G}(x ; \xi)}\right]^{-\beta}}\right]^{-1}, \\
H(x)=-\ln [\bar{F}(x)]=-\ln \left\{1-\exp \left(-\theta^{\beta}\left[\frac{G(x ; \xi)}{\bar{G}(x ; \xi)}\right]^{-\beta}\right)\right\} .
\end{gathered}
$$

\section{Special Models}

A number of new distributions can be deduced as special models from the IW-G family of distributions. Here, four special models, namely; the inverse Weibull Weibull (IWW); the inverse Weibull Pareto (IWP); the inverse Weibull uniform (IWU) and the inverse Weibull Burr XII (IWXII) are introduced.

\subsection{Inverse Weibull Weibull Model}

Suppose, the Weibull cdf and pdf with parameters $\alpha>0, \lambda>0$ are $G(x)=1-$ $e^{-(\lambda x)^{\alpha}} ; x>0$ and $g(x)=\alpha \lambda^{\alpha} x^{\alpha-1} e^{-(\lambda x)^{\alpha}}$. The cdf of a random variable $X$ has the IWW distribution, say $X \sim \operatorname{IWW}(\theta, \beta, \alpha, \lambda)$ is given by

$$
\begin{aligned}
f_{I W W}(x)=\alpha \beta \theta^{\beta} \lambda^{\alpha} x^{\alpha-1} e^{(\lambda x)^{\alpha}}\left(e^{(\lambda x)^{\alpha}}\right. \\
-1)^{-\beta-1} \exp \left[-\theta^{\beta}\left(e^{(\lambda x)^{\alpha}}-1\right)^{-\beta}\right] ; x, \theta, \beta, \alpha, \lambda>0
\end{aligned}
$$

The corresponding distribution function is given by

$$
F_{I W W}(x)=\exp \left[-\theta^{\beta}\left(e^{(\lambda x)^{\alpha}}-1\right)^{-\beta}\right]
$$

The density function (6) reduces to inverse Weibull exponential distribution for $\alpha=1$. Also, for $\alpha=2$, the density function (6) reduces to inverse Weibul Rayleigh distribution. The reliability function and the hazard rate function ( hrf) are obtained as follows

and,

$$
\bar{F}_{I W W}(x)=1-\exp \left[-\theta^{\beta}\left(e^{(\lambda x)^{\alpha}}-1\right)^{-\beta}\right] \text {. }
$$

$$
\begin{gathered}
h_{I W W}(x)=\alpha \beta \theta^{\beta} \lambda^{\alpha} x^{\alpha-1} e^{(\lambda x)^{\alpha}}\left(e^{(\lambda x)^{\alpha}}-1\right)^{-\beta-1} e^{\left[-\theta^{\beta}\left(e^{(\lambda x)^{\alpha}}-1\right)^{-\beta}\right]}[1- \\
\left.e^{\left(-\theta^{\beta}\left(e^{(\lambda x)^{\alpha}}-1\right)^{-\beta}\right)}\right]^{-1}
\end{gathered}
$$

\subsection{Inverse Weibull Pareto Model}

The second model is the inverse Weibull Pareto whose cdf is derived by substituting the following cdf $G(x)=1-\left(\frac{x}{\lambda}\right)^{-\alpha} ; x>\lambda$ in (4) as follows 


$$
F_{I W P}(x)=\exp \left[-\theta^{\beta}\left(\left(\frac{x}{\lambda}\right)^{\alpha}-1\right)^{-\beta}\right] ; \lambda, \alpha, \theta, \beta>0, \lambda<x<\infty .
$$

The probability density function of a random variable $X$ having the IWP distribution, say $X \sim \operatorname{IWP}(\theta, \beta, \alpha, \lambda)$ is given by

$$
f_{I W P}(x)=\alpha \beta \theta^{\beta} \lambda^{-\alpha} x^{\alpha-1}\left(\left(\frac{x}{\lambda}\right)^{\alpha}-1\right)^{-\beta-1} \exp \left[-\theta^{\beta}\left(\left(\frac{x}{\lambda}\right)^{\alpha}-1\right)^{-\beta}\right] \text {. }
$$

Furthermore, the reliability function and hrf are as follows

$$
\bar{F}_{I W P}(x)=1-\exp \left[-\theta^{\beta}\left(\left(\frac{x}{\lambda}\right)^{\alpha}-1\right)^{-\beta}\right]
$$

and,

$$
\begin{gathered}
h_{I W P}(x)=\alpha \beta \theta^{\beta} \lambda^{-\alpha} x^{\alpha-1}\left(\left(\frac{x}{\lambda}\right)^{\alpha}-1\right)^{-\beta-1} e^{-\theta^{\beta}\left(\left(\frac{x}{\lambda}\right)^{\alpha}-1\right)^{-\beta}}[1- \\
\left.e^{-\theta^{\beta}\left(\left(\frac{x}{\lambda}\right)^{\alpha}-1\right)^{-\beta}}\right]^{-1} .
\end{gathered}
$$

\subsection{Inverse Weibull Uniform Model}

Considering the baseline distribution is uniform on the interval $(0, \alpha), \alpha>0$, the cdf of inverse Weibull uniform distribution is as follows

$$
F_{I W U}(x)=\exp \left[-\theta^{\beta}\left(\frac{x}{\alpha-x}\right)^{-\beta}\right] ; \alpha, \theta, \beta>0,0<x<\alpha .
$$

The probability density function of a random variable $X$ having the IWU distribution, say $X \sim \operatorname{IWU}(\theta, \beta, \alpha)$ is given by

$$
f_{I W U}(x)=\alpha \beta \theta^{\beta} x^{-(\beta+1)}(\alpha-x)^{\beta-1} \exp \left[-\theta^{\beta}\left(\frac{x}{\alpha-x}\right)^{-\beta}\right] .
$$

Furthermore, the reliability and hazard rate functions are as follows

$$
\bar{F}_{I W U}(x)=1-\exp \left[-\theta^{\beta}\left(\frac{x}{\alpha-x}\right)^{-\beta}\right],
$$

and,

$$
h_{I W U}(x)=\alpha \beta \theta^{\beta} x^{-(\beta+1)}(\alpha-x)^{\beta-1} e^{-\theta^{\beta}\left(\frac{x}{\alpha-x}\right)^{-\beta}}\left[1-e^{-\theta^{\beta}\left(\frac{x}{\alpha-x}\right)^{-\beta}}\right]^{-1}
$$

\subsection{Inverse Weibull Burr XII Model}

Zimmer et al. (1998) introduced the three-parameter Burr XII (BXII) distribution with the following pdf and cdf 


$$
\begin{gathered}
g(x)=C \alpha \lambda^{-C} x^{C-1}\left[1+\left(\frac{x}{\lambda}\right)^{C}\right]^{-\alpha-1} ; x>0, C, \alpha, \lambda>0, \\
G(x)=1-\left[1+\left(\frac{x}{\lambda}\right)^{C}\right]^{-\alpha} .
\end{gathered}
$$

Hence, the pdf of a random variable $X$ has the inverse Weibull Burr XII distribution, say $X \sim \operatorname{IWBXII}(\theta, \beta, \alpha, \lambda, C)$ is obtained from cdf (4) as follows

$$
F_{I W B X I I}(x)=\exp \left[-\theta^{\beta}\left\{\left(1+\left(\frac{x}{\lambda}\right)^{C}\right)^{\alpha}-1\right\}^{-\beta}\right] ; x>0, \alpha, \theta, \beta, \lambda, C>0 .
$$

The pdf of the IWBXII is given by

$$
\begin{gathered}
f_{I W B X I I}(x)=\alpha \beta C \theta^{\beta} \lambda^{-C} x^{C-1}\left(1+\left(\frac{x}{\lambda}\right)^{C}\right)^{\alpha-1}\left\{\left(1+\left(\frac{x}{\lambda}\right)^{C}\right)^{\alpha}-\right. \\
1\}^{-\beta-1} e^{-\theta^{\beta}\left\{\left(1+\left(\frac{x}{\lambda}\right)^{C}\right)^{\alpha}-1\right\}^{-\beta}} .
\end{gathered}
$$

The reliability and hazard rate functions are

$$
\begin{gathered}
\bar{F}_{I W B X I I}(x)=1-\exp \left[-\theta^{\beta}\left\{\left(1+\left(\frac{x}{\lambda}\right)^{C}\right)^{\alpha}-1\right\}^{-\beta}\right], \\
h_{I W B X I I}(x)=\alpha \beta C \theta^{\beta} \lambda^{-C} x^{C-1}\left(1+\left(\frac{x}{\lambda}\right)^{C}\right)^{\alpha-1}\left\{\left(1+\left(\frac{x}{\lambda}\right)^{C}\right)^{\alpha}-\right. \\
1\}^{-\beta-1} e^{-\theta^{\beta}\left\{\left(1+\left(\frac{x}{\lambda}\right)^{C}\right)^{\alpha}-1\right\}^{-\beta}}\left[1-e^{\left.-\theta^{\beta}\left\{\left(1+\left(\frac{x}{\lambda}\right)^{C}\right)^{\alpha}-1\right\}^{-\beta}\right]^{-1}} .\right.
\end{gathered}
$$

In Figure 1, we display some plots of the pdf of the IWW, IWP, IWU and IWBXII for selected parameter values. Figure 1 reveals that the IWW, IWP, IWU and IWBXII densities generate various shapes such as symmetrical, left-skewed, reversed-J, unimodal and U shaped. 

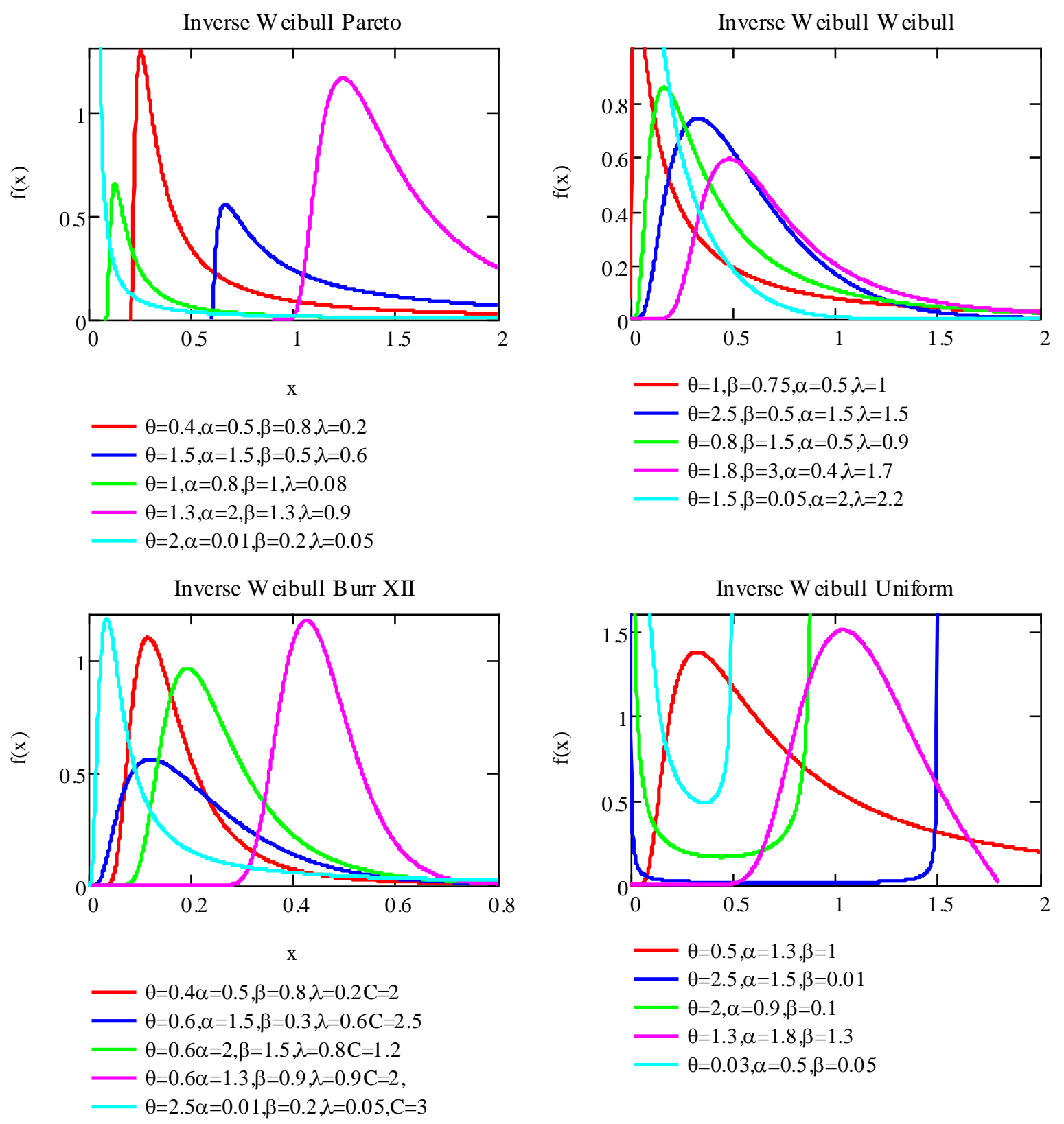

Figure 1: Density plots of the IWW, IWP, IWU and IWBXII models

Plots of the hrf of the IWW, IWP, IWU and IWBXII models are described in Figure 2 for some selected parameter values. From Figure 2, we observe that these models can produce hazard rate shapes such as constant, increasing, decreasing, and upside-down bathtub. This fact implies that the IW-G family can be very useful for fitting data sets with various shapes. 

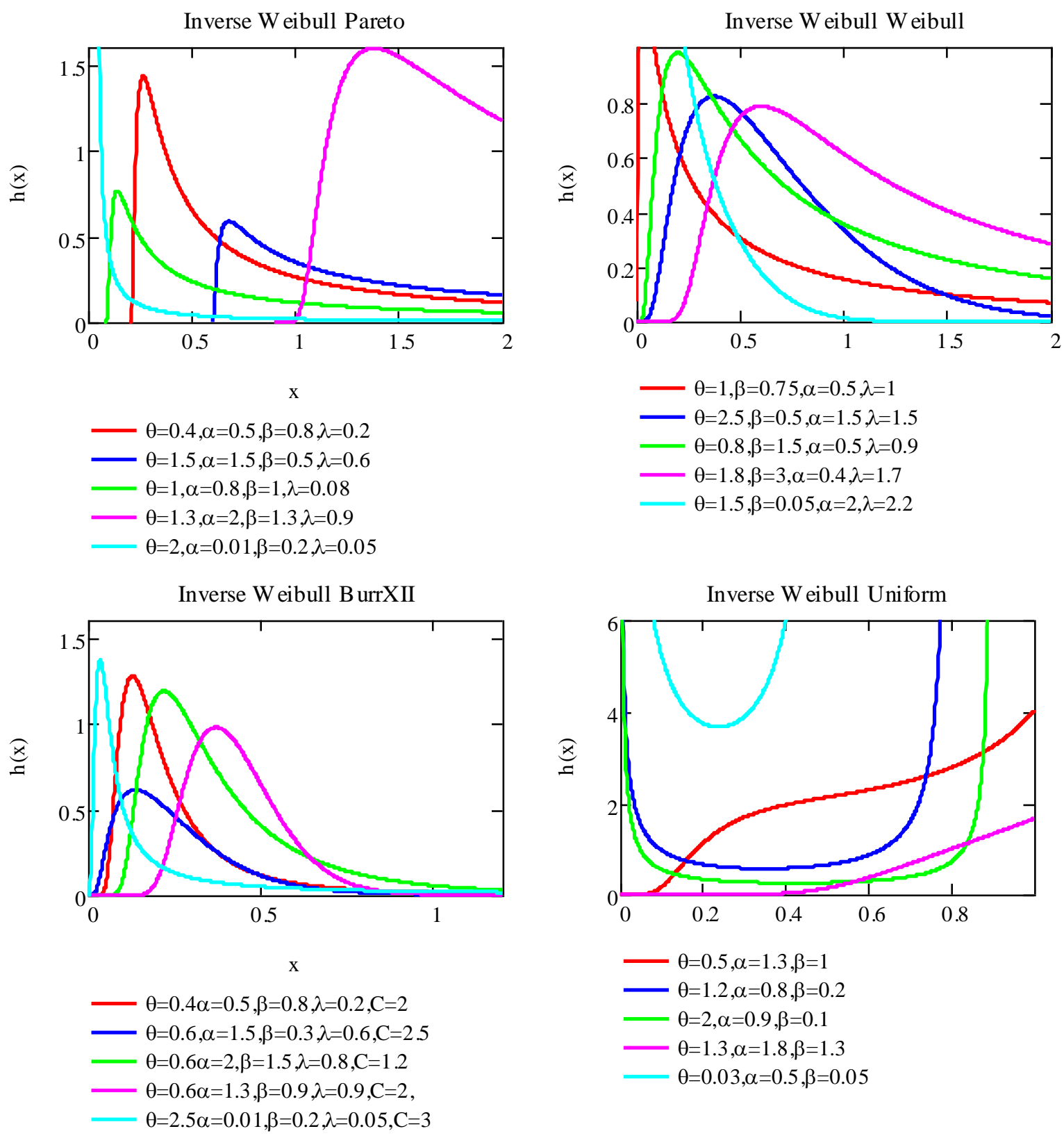

Figure 2: Hazard rate function plots of the $I W W, I W P, I W U$ and $I W B X I I$ models

\section{Mathematical Properties}

In this section, we provide some main mathematical properties of the IW-G family. 


\subsection{Useful Expansions}

Some mathematical properties of the IW-G family can be confirmed through an algebraic expansion which is more efficient than computing those directly by numerical integration of its density function. Here two important expansions are deduced for the IW $\mathrm{G}$ pdf and cdf using mixture forms of exponentiated-G (Exp-G) distribution.

Since, the power series for the exponential function in pdf (5) can be written as follows:

$$
e^{-\theta^{\beta}\left(\frac{G(x ; \xi)}{\bar{G}(x ; \xi)}\right)^{-\beta}}=\sum_{i=0}^{\infty} \frac{(-1)^{i} \theta^{i \beta}}{i !}\left(\frac{G(x ; \xi)}{\bar{G}(x ; \xi)}\right)^{-\beta i}
$$

Inserting expansion (7) in pdf (5), then we have

$$
f(x)=\beta \sum_{i=0}^{\infty} \frac{(-1)^{i} \theta^{\beta(i+1)} g(x ; \xi)(G(x ; \xi))^{-(\beta i+\beta+1)}}{i !(\bar{G}(x ; \xi))^{-(\beta i+\beta+1)}}
$$

It is well-known that, if $\beta>0$ is real non integer and $|z|<1$, the generalized binomial theorem is written as follows

$$
(1-z)^{\beta-1}=\sum_{j=0}^{\infty}(-1)^{j}\left(\begin{array}{c}
\beta-1 \\
j
\end{array}\right) z^{j}
$$

Then, by applying the binomial theorem (9) in (8), the IW-G pdf, where $\beta$ is real non integer becomes

$$
f(x)=\sum_{i, j=0}^{\infty}\left(\begin{array}{c}
\beta i+\beta-1 \\
j
\end{array}\right) \frac{(-1)^{i+j} \beta \theta^{\beta(i+1)}}{i !} g(x ; \xi)(G(x ; \xi))^{j-\beta(i+1)-1} .
$$

Hence, IW-G density function can be expressed as an infinite mixture of Exp-G density functions as follows

$$
\left.\begin{array}{c}
f(x)=\sum_{i, j=0}^{\infty} \eta_{i, j} W_{k}(x ; \xi) ; \quad x>0, \\
\eta_{i, j}=\left(\begin{array}{c}
\beta i+\beta-1 \\
j
\end{array}\right) \frac{(-1)^{i+j} \beta \theta^{\beta(i+1)}}{i ! k}, k=j-\beta(i+1)
\end{array}\right\}
$$

where, $W_{k}(x ; \xi)=k g(x ; \xi)(G(x ; \xi))^{k-1}$ denotes the 'exp-G' pdf with power parameter $k$.

Additionally, using the exponential expansion for $[F(x)]^{s}$, where sis a positive integer, becomes

$$
[F(x)]^{s}=\sum_{m=0}^{\infty} \frac{(-1)^{m} s^{m} \theta^{\beta m}}{m !}\left(\frac{G(x ; \xi)}{\bar{G}(x ; \xi)}\right)^{-\beta m}
$$

Using the binomial expansion (9) in (11) where $\beta$ is a real non integer, leads to :

$$
[F(x)]^{s}=\sum_{m, l=0}^{\infty} \psi_{m, l}(G(x ; \xi))^{l-\beta m}
$$


where, $\psi_{m, l}=\frac{(-1)^{m+l} s^{m} \theta^{\beta m}}{m !}\left(\begin{array}{c}\beta m+1 \\ l\end{array}\right)$.

\subsection{Quantile Function}

In this subsection, the quantile function of a random variable $X$ has the IW-G distribution is derived. More specifically, the quantile function for the IWP model is obtained. Further, the skewness and kurtosis based on quantile function for IWP model are discussed.

The quantile function of IW-G family, say $x=Q(x)=F^{-1}(u)$ can be obtained by inverting (4) as follows

$$
x=Q(x)=F^{-1}(u)=G^{-1}\left\{\left(-\theta^{-\beta} \ln (u)\right)^{-1 / \beta}\left[1+\left(-\theta^{-\beta} \ln (u)\right)^{-1 / \beta}\right]\right\} .
$$

Solving this equation for $Q(x)$ gives the quantile function.

Example 1: Consider the IWP distribution discussed in subsection (3.2). The quantile function of IWP is obtained as follows

$$
x=Q(x)=\lambda\left[1+\theta(-\ln (u))^{-1 / \beta}\right]^{1 / \alpha} .
$$

Kenney and Keeping (1962) proposed skewness based on quartiles called the Bowley skewness which is defined as follows

$$
B=\frac{Q\left(\frac{3}{4}\right)-2 Q\left(\frac{1}{2}\right)+Q\left(\frac{1}{4}\right)}{Q\left(\frac{3}{4}\right)-Q\left(\frac{1}{4}\right)} .
$$

Further, the Moors kurtosis (see Moors (1988)) based on octiles is defined as follows

$$
M=\frac{Q\left(\frac{7}{8}\right)-Q\left(\frac{5}{8}\right)+Q\left(\frac{3}{8}\right)-Q\left(\frac{1}{8}\right)}{Q\left(\frac{6}{8}\right)-Q\left(\frac{2}{8}\right)} .
$$

where, $Q($.$) denotes the quantile function. Figure 3$ gives plots of the skewness and kurtosis for some choices of the parameter $\beta$ as function of $\alpha$. These plots indicate that the skewness and kurtosis decrease when $\beta$ increases for fixed $\alpha$.

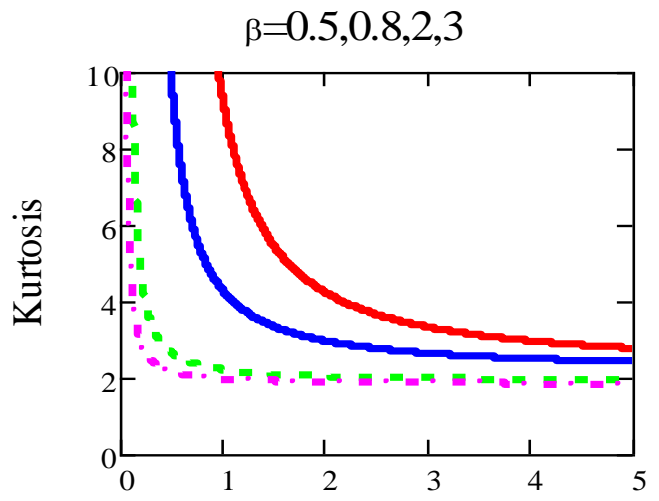

$\alpha$

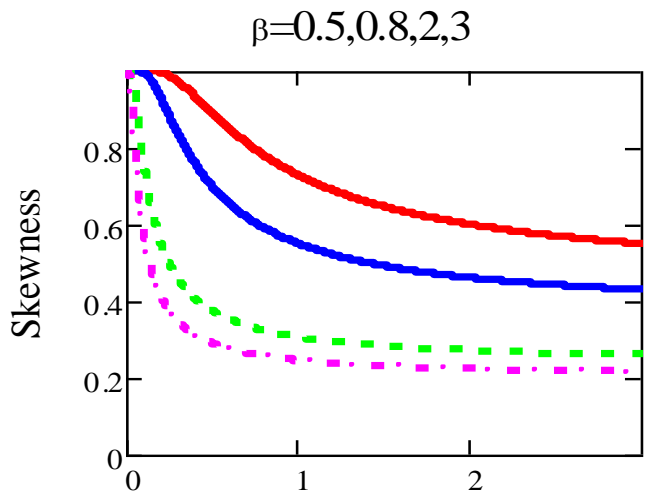

Figure 3: Plots of skewness and kurtosis for IWP distribution based on quantile. 


\subsection{Moments}

Most of the necessary characteristics and features of a distribution can be studied through its moments. Here, the moments of a random variable $X$ has the IW-G are derived. More specifically, the $r$ th moment for the IWU model is obtained.

The $r$ th moment of $X$ about the origin is derived from (10) as follows

$$
\mu_{r}^{\prime}=\sum_{i, j=0}^{\infty} \eta_{i, j} \int_{-\infty}^{\infty} x^{r} W_{k}(x ; \xi) d x, \quad r=1,2, \ldots
$$

Furthermore, the moment generating function of $X$ is

$$
M_{X}(t)=\sum_{r=0}^{\infty} \frac{t^{r}}{r !} \mu_{r}^{\prime}=\sum_{r=0}^{\infty} \sum_{i, j=0}^{\infty} \frac{t^{r}}{r !} \eta_{i, j} \int_{-\infty}^{\infty} x^{r} W_{k}(x ; \xi) d x .
$$

Example 2: Consider the IWU distribution discussed in subsection (3.3). The rth moment of IWU can be obtained from (13) with pdf and cdf as defined in subsection (3.3) as follows

$$
\mu_{r}^{\prime}=\sum_{i, j=0}^{\infty} \eta_{i, j} \frac{j-\beta(i+1)}{r+(j-\beta(i+1))} \alpha^{r} .
$$

In particular, the mean and variance of the IWU distribution are obtained, respectively, as follows:

$$
\begin{gathered}
E(X)=\sum_{i, j=0}^{\infty} \eta_{i, j} \frac{j-\beta(i+1)}{(j-\beta(i+1))+1} \alpha \\
\operatorname{Var}(X)=\sum_{i, j=0}^{\infty} \eta_{i, j} \frac{j-\beta(i+1)}{(j-\beta(i+1))+2} \alpha^{2}-\left[\sum_{i, j=0}^{\infty} \eta_{i, j} \frac{j-\beta(i+1)}{(j-\beta(i+1))+1} \alpha\right]^{2} .
\end{gathered}
$$

\subsection{The probability Weighted Moments}

The probability-weighted moments (PWMs) method of estimation has been proposed by Greenwood et al. (1979) for distribution expressible in inverse form. For a random variable $X$ the PWMs, denoted by $\tau_{r, s}$, can be calculated through the following relation

$$
\tau_{r, s}=E\left[X^{r} F(x)^{s}\right]=\int_{-\infty}^{\infty} x^{r} f(x)[F(x)]^{s} d x
$$

The PWMs of IW-G family is obtained by inserting (10) and (12) into (14) as follows

$$
\tau_{r, s}=\sum_{i, j=0}^{\infty} \sum_{m, l=0}^{\infty} \zeta_{i, j, k, m, l} \int_{-\infty}^{\infty} x^{r} g(x ; \xi)[G(x ; \xi)]^{l-\beta m+k-1} d x,
$$

where, $\zeta_{i, j, k, m, l}=\psi_{m, l} \eta_{i, j} k$ and $k=j-\beta(i+1)$.

Example 3: Consider the IWP distribution discussed in subsection (3.2). The PWMs of IWP distribution can be obtained as follows

$$
\begin{gathered}
\tau_{r, s}=\sum_{i, j, m, l=0}^{\infty} \zeta_{i, j, k, m, l} \int_{\lambda}^{\infty} x^{r} \frac{\alpha}{\lambda}\left(\frac{x}{\lambda}\right)^{-\alpha-1}\left[1-\left(\frac{x}{\lambda}\right)^{-\alpha}\right]^{l-\beta m+k-1} d x, \\
\tau_{r, s}=\sum_{i, j, m, l=0}^{\infty} \zeta_{i, j, k, m, l} \lambda^{r} B\left(1-\frac{r}{\alpha}, l-\beta m+k\right) .
\end{gathered}
$$

where, $B(.,$.$) stands for beta function and k=j-\beta(i+1)$. 


\subsection{The Mean Deviation}

In statistics, the mean deviation about the mean and mean deviation about the median measure the amount of scattering in a population. For random variable $X$ with pdf $f(x)$, cdf $F(x)$, the mean deviation about the mean and mean deviation about the median, are defined by

$$
\delta_{1}(X)=2 \mu F(\mu)-2 T(\mu) \text { and } \delta_{2}(X)=\mu-2 T(M)
$$

where, $\mu=E(X), M=$ Median $(X)$, and $T(q)=\int_{-\infty}^{q} x f(x) d x$ which is the first incomplete moment. Another application of the first incomplete moment refers to the Bonferroni and Lorenz curves. These curves are very useful in economics, reliability, demography, insurance and medicine.

Example 4: Consider the IWU distribution discussed in subsection (3.2). The first incomplete moment of IWU distribution can be obtained as follows

$$
T(q)=\sum_{i, j=0}^{\infty} \eta_{i, j} \int_{0}^{q} x k\left(\frac{1}{\alpha}\right)\left(\frac{x}{\alpha}\right)^{k-1} d x=\sum_{i, j=0}^{\infty} \eta_{i, j} \frac{k q}{k+1},
$$

where, $\eta_{i, j}=\left(\begin{array}{c}\beta i+\beta-1 \\ j\end{array}\right) \frac{(-1)^{i+j} \beta \theta^{\beta(i+1)}}{i ! k}$ and $k=j-\beta(i+1)$.

\subsection{Order Statistics}

Suppose $X_{1}, X_{2}, \ldots, X_{n}$ be independent and identically distributed (i.i.d) random variables with their corresponding continuous distribution function $F(x)$. Let $X_{1: n}<$ $X_{2: n}<\ldots<X_{n: n}$ the corresponding ordered random sample from a population of size $n$. According to David (1981), the pdf of the $r$ th order statistic, is defined by

$$
f_{r: n}(x)=\frac{1}{B(r, n-r+1)} \sum_{v=0}^{n-r}(-1)^{r}\left(\begin{array}{c}
n-r \\
v
\end{array}\right) f(x) F(x)^{v+r-1}
$$

The pdf of the $r$ th order statistic for IW-G family is derived by substituting (10) and (12) in (15), replacing $s$ with $v+r-1$,

$$
\left.\begin{array}{l}
f_{r: n}(x)= \\
\frac{1}{B(r, n-r+1)} \sum_{v=0}^{n-r} \sum_{i, j, m, l=0}^{\infty}(-1)^{r}\left(\begin{array}{c}
n-r \\
v
\end{array}\right) \eta_{i, j} \psi_{m, l} g(x ; \xi)[G(x ; \xi)]^{l-\beta m+j-\beta(i+1)-1} \\
\quad f_{r: n}(x)=\sum_{v=0}^{n-r} \sum_{i, j, m, l=0}^{\infty} N_{v, i, j, m, l} W_{k_{1}}^{*}(x ; \xi), \\
N_{v, i, j, m, l}=\frac{k \theta^{\beta m}(v+r-1)^{m}(-1)^{r+m+l}}{B(r, n-r+1) k_{1} m !}\left(\begin{array}{c}
n-r \\
v
\end{array}\right)\left(\begin{array}{c}
\beta m+1 \\
l
\end{array}\right) \eta_{i, j}, k_{1}=l-\beta m+j-\beta(i+1)
\end{array}\right\}
$$

where, $W_{k_{1}}^{*}(x ; \xi)$ denotes the 'exp-G' pdf with power parameter $k_{1}$. In particular, the pdf of the smallest order statistics $X_{1: n}$ is obtained from (16), by substituting $r=1$, as follows

$$
f_{1: n}(x)=\sum_{v=0}^{n-1} \sum_{i, j, m, l=0}^{\infty} M_{v, i, j, m, l} W_{k_{1}}^{*}(x ; \xi),
$$

where, $M_{v, i, j, m, l}=\frac{n k(-1)^{m+l+1} \theta^{\beta m} v^{m}}{k_{1} m !}\left(\begin{array}{c}n-1 \\ v\end{array}\right)\left(\begin{array}{c}\beta m+1 \\ l\end{array}\right) \eta_{i, j}$. 
Also, the pdf of the largest order statistics $X_{n: n}$ is obtained by substituting $r=n$ in (16),

$$
f_{n: n}(x)=\sum_{i, j, m, l=0}^{\infty} L_{i, j, m, l} W_{k_{1}}^{*}(x ; \xi),
$$

where, $L_{i, j, m, l}=\frac{n k \theta^{\beta m}(-1)^{n+m+l}(v+n-1)^{m}}{k_{1} m !}\left(\begin{array}{c}\beta m+1 \\ l\end{array}\right) \eta_{i, j}$.

\subsection{Rènyi Entropy}

Entropy is a measure of variation or uncertainty of a random variable $X$ (Rènyi, 1961). The Rènyi entropy of a random variable is defined by

$$
I_{\delta}(X)=\frac{1}{1-\delta} \log \int_{-\infty}^{\infty} f(x)^{\delta} \quad d x, \delta>0 \text { and } \delta \neq 1
$$

By applying the binomial theory (9) and exponential expansion, then the pdf $f(x)^{\delta}$ can be expressed as follows

$$
=\sum_{i, j=0}^{f(x)^{\delta}} \frac{(-1)^{i+j} \delta^{i} \theta^{\beta(\delta+i)}}{i !}\left(\begin{array}{c}
\delta(\beta+\beta i-1)+1 \\
j
\end{array}\right)(g(x ; \xi))^{\delta}(G(x ; \xi))^{j-\delta(\beta+\beta i+1)}
$$

Inserting (18) in (17), then the Rènyi entropy of the family is as follows

$$
\begin{aligned}
& I_{\delta}(X)=\frac{1}{1-\delta} \log \sum_{i, j=0}^{\infty} \frac{(-1)^{i+j} \delta^{i} \theta^{\beta(\delta+i)}}{i !}\left(\begin{array}{c}
\delta(\beta+\beta i-1)+1 \\
j
\end{array}\right) \mathfrak{I}(\delta, i, j), \\
& \text { where, } \mathfrak{J}(\delta, i, j)=\int_{-\infty}^{\infty}(g(x ; \xi))^{\delta}(G(x ; \xi))^{j-\delta(\beta+\beta i+1)} d x
\end{aligned}
$$

Example 5:Consider again, the IWP distribution discussed in subsection (3.2). The Rènyi entropy of IWP distribution can be derived as follows

$$
\begin{gathered}
I_{\delta}(X)=\frac{1}{1-\delta} \log \left[\sum _ { i , j = 0 } ^ { \infty } \frac { ( - 1 ) ^ { i + j } \delta ^ { i } \theta ^ { \beta ( \delta + i ) } } { i ! } ( \begin{array} { c } 
{ \delta ( \beta + \beta i - 1 ) } \\
{ j }
\end{array} ) ( \frac { \alpha } { \lambda } ) ^ { \delta - 1 } B \left(\frac{\delta(\alpha+1)-1}{\alpha}, j-\right.\right. \\
\delta(\beta+\beta i-1)+1)] .
\end{gathered}
$$

\section{Estimation of Parameters}

This section concerns with the maximum likelihood estimates (MLEs) of the unknown parameters for the new family based on complete samples. Let $X_{1}, X_{2}, \ldots, X_{n}$ be a simple random sample from pdf (5) with set of parameters $\Theta \equiv(\beta, \theta, \xi)$. The log-likelihood function, denoted by $\ln L$, based on the observed random sample of size $n$ from density (5) is given by:

$$
\begin{aligned}
\ln L= & n \ln \beta+n \beta \ln \theta+\sum_{i=1}^{n} \ln g\left(x_{i}, \xi\right)-2 \sum_{i=1}^{n} \ln \bar{G}\left(x_{i}, \xi\right)- \\
& (\beta+1) \sum_{i=1}^{n} \ln \left[Z\left(x_{i}, \xi\right)\right]-\theta^{\beta} \sum_{i=1}^{n}\left[Z\left(x_{i}, \xi\right)\right]^{-\beta},
\end{aligned}
$$

where, $Z\left(x_{i}, \xi\right)=\frac{G\left(x_{i}, \xi\right)}{\bar{G}\left(x_{i}, \xi\right)}$. 
The elements of the score function $U(\Theta)=\left(U_{\theta}, U_{\beta}, U_{\xi}\right)$ are given by $U_{\theta}=\frac{n \beta}{\theta}-$ $\beta \theta^{\beta-1} \sum_{i=1}^{n}\left[Z\left(x_{i}, \xi\right)\right]^{-\beta}$,

$$
\begin{gathered}
U_{\beta}=\frac{n}{\beta}+n \ln \theta-\sum_{i=1}^{n} \ln \left[Z\left(x_{i}, \xi\right)\right]+\theta^{\beta} \sum_{i=1}^{n}\left[Z\left(x_{i}, \xi\right)\right]^{-\beta} \ln \left[Z\left(x_{i}, \xi\right)\right]- \\
\theta^{\beta} \ln \theta \sum_{i=1}^{n}\left[Z\left(x_{i}, \xi\right)\right]^{-\beta},
\end{gathered}
$$

and

$$
\begin{gathered}
U_{\xi}=\sum_{i=1}^{n} \frac{\partial g\left(x_{i}, \xi\right) / \partial \xi_{k}}{g\left(x_{i}, \xi\right)}-2 \sum_{i=1}^{n} \frac{\partial \bar{G}\left(x_{i}, \xi\right) / \partial \xi_{k}}{\bar{G}\left(x_{i}, \xi\right)}-(\beta+1) \sum_{i=1}^{n} \frac{\partial Z\left(x_{i}, \xi\right) / \partial \xi_{k}}{Z\left(x_{i}, \xi\right)} \\
+\beta \theta^{\beta} \sum_{i=1}^{n}\left[Z\left(x_{i}, \xi\right)\right]^{-\beta-1} \frac{\partial z\left(x_{i}, \xi\right)}{\partial \xi_{k}}
\end{gathered}
$$

Setting $U_{\theta}, U_{\beta}$ and $U_{\xi}$ equal to zero and solving the equations simultaneously the MLE, say $\widehat{\Theta} \equiv(\hat{\beta}, \hat{\theta}, \hat{\xi})^{T}$ of $\Theta \equiv(\beta, \theta, \xi)^{T}$ are obtained. Further the resulting equations cannot be solved analytically, so some software's can be used to solve them numerically.

For interval estimation of the model parameters, we obtain the $3 \times 3$ observed information matrix $I=I(\Theta)$ (for $r, s=\beta, \theta, \xi$ ), whose elements are listed in Appendix. Under standard regularity conditions, the multivariate normal $N_{3}\left(0, I^{-1}(\widehat{\Theta})\right)$ distribution is used to construct approximate confidence intervals for the parameters. The approximate $100(1-\gamma) \%$ two sided confidence intervals for $\theta, \beta, \xi$ are respectively, given by:

$$
\hat{\alpha} \pm Z_{\gamma / 2} \sqrt{\operatorname{Var}(\hat{\alpha})}, \hat{\beta} \pm Z_{\gamma / 2} \sqrt{\operatorname{Var}(\hat{\beta})} \text { and } \hat{\xi} \pm Z_{\gamma / 2} \sqrt{\operatorname{Var}(\hat{\xi})}
$$

Here, $Z_{\gamma / 2}$ is the upper $\gamma / 2^{\text {th }}$ percentile of the standard normal distribution and $\operatorname{Var}($.$) 's denote the diagonal elements of I^{-1}(\widehat{\Theta})$ corresponding to the model parameters.

\section{Applications To Real Data}

The flexibility of IWW as especial model from IW-G family is examined using two real data sets. The superiority of IWW is clarified as compared with some main four models; additive Weibull (AW; Almalki and Yuan (2013)), new modified Weibull (NMW; Doostmoradi et al. (2014)), Weibull Weibull (WW; Bourguignon et al. (2014)) and Weibull (W).

For both data sets, the unknown parameters of each distribution are estimated by the maximum-likelihood method. The model selection is carried out using KolmogorovSmirnov (K-S) statistic and corresponding P-value, $-2 \log$-likelihood function $(-2 \ln L)$, Akaike information criterion (AIC), the correct Akaike information criterion (CAIC), Bayesian information criterion (BIC) and Hannan-Quinn information criterion (HQIC). However, the better distribution corresponds to the smaller values of AIC, CAIC, BIC, HQIC, K-S criteria and largest values of P-value. Furthermore, we plot the histogram for each data set and the estimated pdf of the four models. Moreover, plots of empirical cdf of the data sets and estimated cdf of four models are displayed. 
Example 6.1: The first data set is provided in Murthy et al. (2004) about time between failures for repairable item. The data are listed as the following:

$1.43,0.11,0.71,0.77,2.63,1.49,3.46,2.46,0.59,0.74,1.23,0.94,4.36,0.40,1.74,4.73$, $2.23,0.45,0.70,1.06,1.46,0.30,1.82,2.37,0.63,1.23,1.24,1.97,1.86,1.17$.

In Table (1), we list the values of AIC, CAIC, BIC, HQIC, K-S and the P-value statistics. We observe that the IWW model has the smallest AIC, CAIC, BIC, HQIC, K-S values, and has the largest P-value as compared with those values of the other models. So, the IWW model seems to be a very competitive model to this data. More information is provided by a visual comparison of the histogram and estimated cumulative of the data with the fitted models as shown in Figure 4. It is clear from Figure 4 that the IWW distribution provides a better fit than the other competitive models.

Table (1): Model Selection Criteria for the First Data Set

\begin{tabular}{|c|c|c|c|c|c|c|c|}
\hline Model & -2InL & $\boldsymbol{A I C}$ & $\boldsymbol{B I C}$ & $\boldsymbol{C A I C}$ & $\boldsymbol{H Q I C}$ & $\boldsymbol{K}-\boldsymbol{S}$ & P-value \\
\hline $\boldsymbol{I W} \boldsymbol{W}$ & 79.21 & 87.21 & 92.815 & 88.81 & 89.003 & 0.070 & 998.0 \\
\hline $\boldsymbol{N M} \boldsymbol{W}$ & 242.501 & 250.051 & 255.656 & 251.651 & 251.845 & 0.942 & 0.000 \\
\hline $\boldsymbol{A} \boldsymbol{W}$ & 159.642 & 167.642 & 173.246 & 169.242 & 169.435 & 0.283 & 0.017 \\
\hline $\boldsymbol{W} \boldsymbol{W}$ & 80.276 & 88.276 & 93.88 & 89.876 & 90.069 & 0.086 & 0.978 \\
\hline $\boldsymbol{W}$ & 92.751 & 96.751 & 99.554 & 97.196 & 97.648 & 0.134 & 0.655 \\
\hline
\end{tabular}
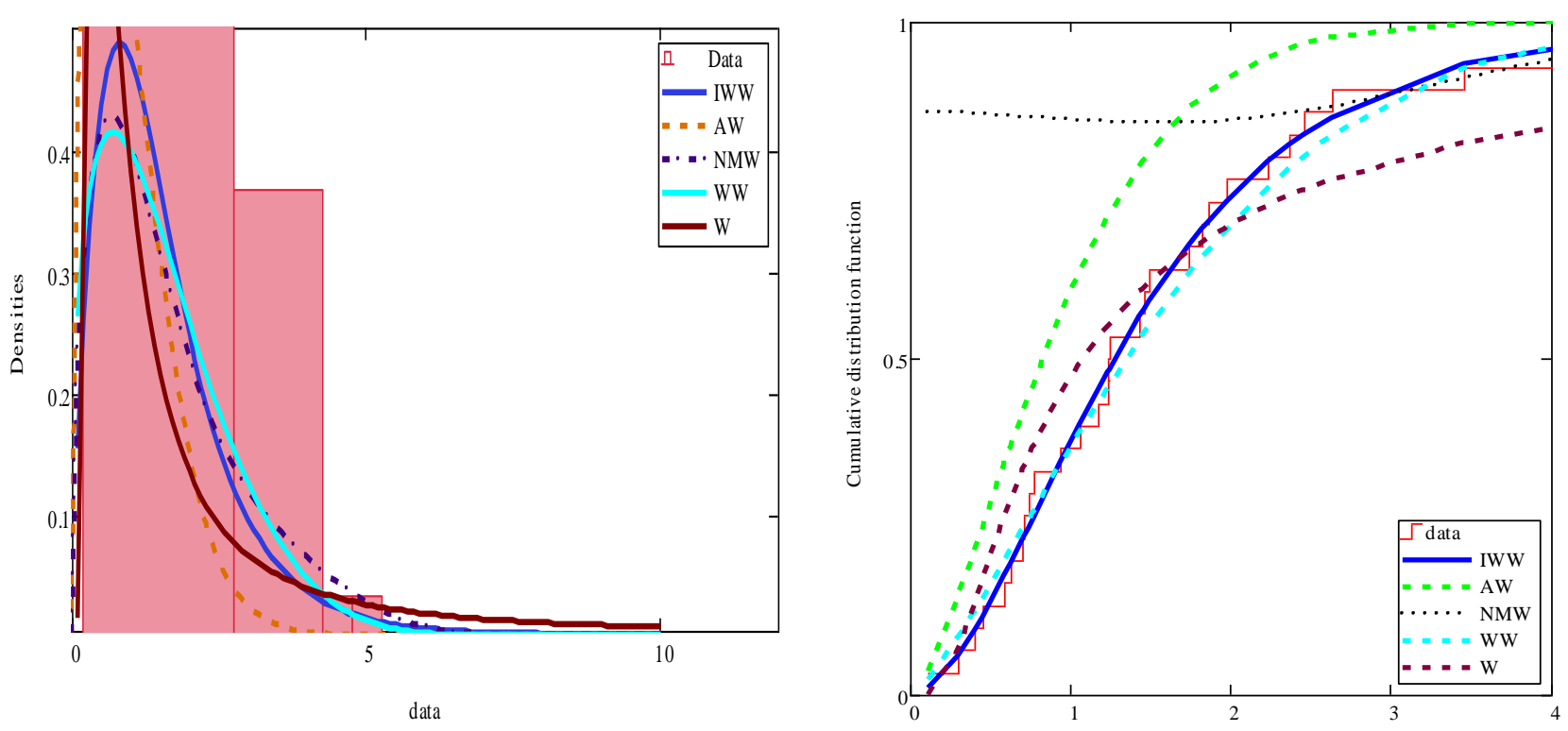

Figure 4: Estimated densities and estimated distributions of models for the first data set 
Example 6.2: The second data set represents 34 observations of the vinyl chloride data obtained from clean up gradient ground -water monitoring wells in $\mathrm{mg} / \mathrm{L}$. The data are obtained from Bhaumik et al. (2009) and recorded as follows

$5.1,1.2,1.3,0.6,0.5,2.4,0.5,1.1,8.0,0.8,0.4,0.6,0.9,0.4,2.0,0.5,5.3,3.2,2.7,2.9$, $2.5,2.3,1.0,0.2,0.1,0.1,1.8,0.9,2.0,4.0,6.8,1.2,0.4,0.2$.

Results in Table 2, indicate that the IWW model is more suitable than the other competitive models for this data set based on the selected criteria. Further, it is clear from Figure 5 that the IWW distribution provides a better fit and therefore be one of the best models for this data set.

Table (2): Model Selection Criteria for the Second Data Set

\begin{tabular}{|c|c|c|c|c|c|c|c|}
\hline Model & $\mathbf{- 2 \operatorname { l n L }}$ & $\boldsymbol{A I C}$ & $\boldsymbol{B I C}$ & $\boldsymbol{C A I C}$ & $\boldsymbol{H Q I C}$ & $\boldsymbol{K}-\boldsymbol{S}$ & P-value \\
\hline $\boldsymbol{I} \boldsymbol{W}$ & 108.470 & 116.47 & 122.576 & 117.850 & 118.552 & 0.087 & 959.0 \\
\hline $\boldsymbol{N} \boldsymbol{W} \boldsymbol{W}$ & 314.807 & 322.807 & 328.912 & 324.186 & 324.889 & 1 & 0.000 \\
\hline $\boldsymbol{A} \boldsymbol{W}$ & 221.798 & 229.798 & 235.904 & 231.178 & 231.881 & 0.982 & 0.018 \\
\hline $\boldsymbol{W} \boldsymbol{W}$ & 111.160 & 119.160 & 125.265 & 120.539 & 121.242 & 0.094 & 0.922 \\
\hline $\boldsymbol{W}$ & 117.253 & 121.253 & 124.306 & 121.640 & 122.294 & 0.113 & 0.775 \\
\hline
\end{tabular}
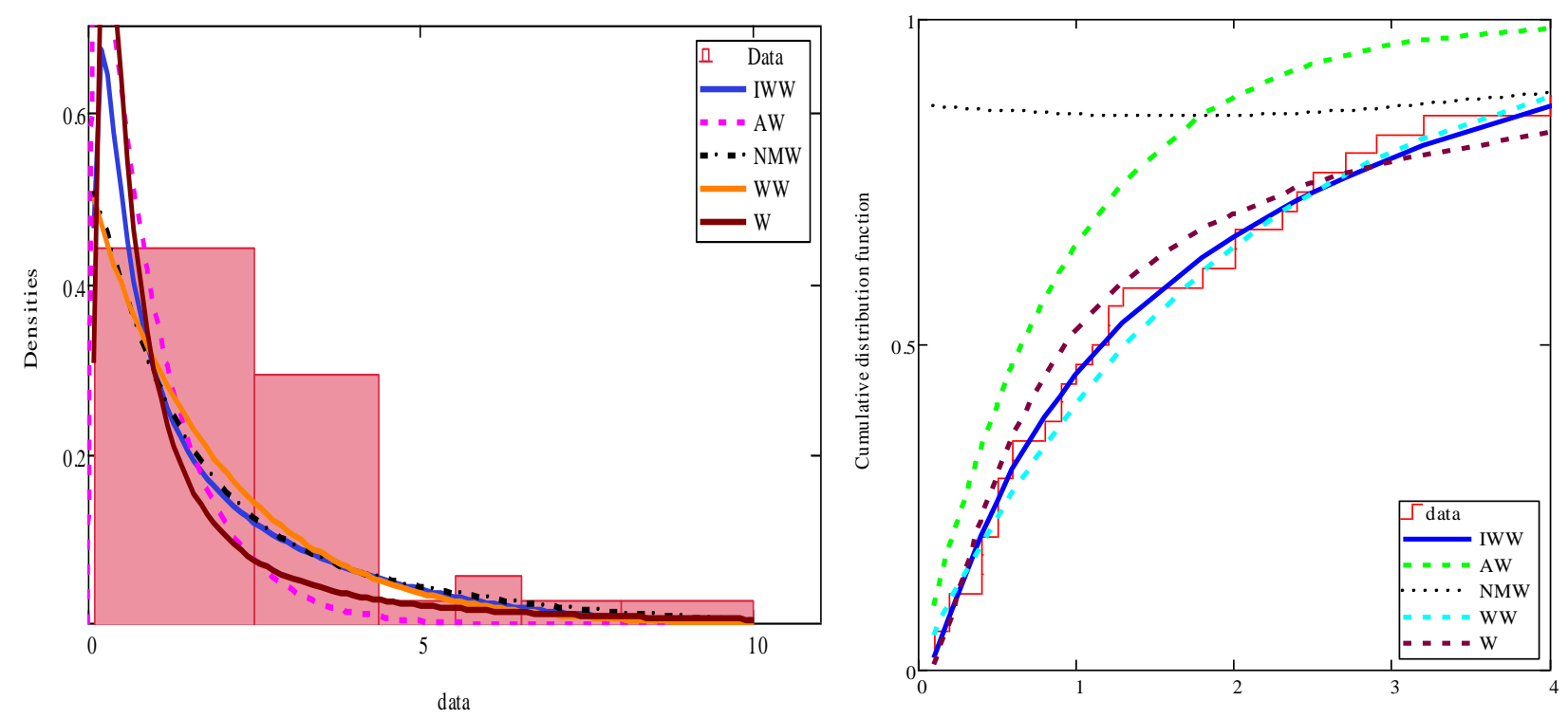

Figure 5: Estimated densities and estimated distributions of models for the second data set. 


\section{Concluding Remarks}

In this paper, we introduce a new family of univariate distribution based on the inverse Weibull distribution as a new generator. Many new sub-models are obtained and four special models are provided. The IW-G density function can be expressed as a mixture of exponentiated-G distribution functions. Mathematical properties of the IW-G family are derived. We give explicit closed form expressions for the moments, probability weighted, entropy and distribution of order statistics. The maximum likelihood method of estimation is employed to derive the model parameters and the observed Fisher information matrix is obtained. We fit IWW distribution as special sub-model to two real data sets in order to explain the flexibility of this new family. We hope this new generation may attract wider applications in many areas. 


\section{References}

[1] Alexander C., Cordeiro G. M., Ortega E.M.M., and Sarabia, J. M. (2012). Generalized betagenerated distributions. Computational Statistics \& Data Analysis, 56:1880-1897.

[2] Almalki, S. J., and Yuan, J. (2013). A new modified Weibull distribution. Reliability Engineering and System Safety, 111: 164-170.

[3] Alzaatreh, A., Lee, C., and Famoye F. (2013). A new method for generating families of distributions. Metron, 71: 63-79.

[4] Bhaumik, D. K., Kapur K. G. and Gibbons, R. D. (2009). Testing parameters of a gamma distribution for small samples. Technometrics, 51(3): 326-334.

[5] Bourguignon, M., Silva, R. B., and Cordeiro, G. M. (2014). The Weibull-G family of probability distributions. Journal of Data Science, 12: 53-68.

[6] Calabria, R., and Pulcini, G., (1990). On the maximum likelihood and least-squares estimation in the inverse Weibull distribution. Statistica Applicata, 2 (1): 53-63.

[7] Calabria, R., and Pulcini, G., (1994). Bayes 2-sample prediction for the inverse Weibull distribution. Communication of Statistics Theory \&Methods, 23 (6): 1811-1824.

[8] Corderio G. M., and de Castro, M. (2011). A new family of generalized distributions. Journal of Statistical Computation and Simulation, 81: 883-893.

[9] Cordeiro, G. M., Ortega, E. M. M., and da Cunha, D. C. C. (2013). The exponentiated generalized class of distributions. Journal of Data Science, 11: 1-27.

[10] David, H. A. (1981). Order Statistics, 2nd ed., Wiley, New York.

[11] Doostmoradi, A., Zadkarami, M. R. and Roshani Sheykhabad, A. (2014) A new modified Weibull distribution and its applications. Journal of Statistical Research, 11: 97-118.

[12] Eugene, N., Lee, C., and Famoye F. (2002). Beta-normal distribution and its applications. Communication in Statistics - Theory \& Methods, 31: 497-512.

[13] Greenwood, J. A., Landwehr, J. M., Matalas, N. C., and Wallis, J. R. (1979). Probability weighted moments: definition and relation to parameters of several distributions expressible in inverse form, Water Resources Research, 15(5): 1049-1054.

[14] Haq, M. A. and Elgarhy, M., (2018). Odds Frèchet-G family of probability distributions. Journal of Statistics Applications \& Probability, 7(1): 185-201.

[15] Hassan, A. S., and Al-Thobety, A. K. (2012). Optimal design of failure step stress partially accelerated life tests with type II censored inverted Weibull data. International Journal of Engineering Research and Applications, 2 (3): 3242-3253. 
[16] Hassan, A. S., and Elgarhy, M., (2016 a). Kumaraswamy Weibull-generated family of distributions with applications. Advances and Applications in Statistics, 48: 205-239.

[17] Hassan, A.S. and Elgarhy, M., (2016 b). A new family of exponentiated Weibull-generated distributions. International Journals of Mathematics and Its Applications, 4: 135-148.

[18] Hassan, A. S., and Hemeda, S. E. (2016). The additive Weibull-G family of probability distributions. International Journals of Mathematics and Its Applications, 4: 151-164.

[19] Hassan, A. S., Assar, M. S. and Zaky, A. N. (2015). Constant-stress partially accelerated life tests for inverted Weibull distribution with multiple censored data. International Journal of Advanced Statistics and Probability, 3 (1): 72-82.

[20] Hassan, A. S., Elgarhy, M., and Shakil, M. (2017a). Type II half logistic family of distributions with applications. Pakistan Journal of Statistics \&Operation Research, 13(2): 245264.

[21] Hassan, A. S., Hemeda, S. E., Maiti, S.S. and Pramanik, S. (2017 b). The generalized additive Weibull-G family of probability distributions. International Journal of Statistics and Probability, 6(5): 65-83.

[22] Hassan, A. S., and Nassr, S. G. (2018). Power Lindley- G family. Annals of Data Science, $1-22$

[23] Jiang, R., Murthy, D. N. P., and Ping, J. I. (2001). Models involving two inverse Weibull distributions. Reliability Engineering System Safety, 73 (1): 73-81.

[24] Keller, A. Z., and Kamath, A. R. R., (1982). Alternative reliability models for mechanical systems. Third International Conference on Reliability and Maintainability, Toulse, France.

[25] Kenney, J. F. and Keeping, E. (1962). Mathematics of Statistics. D. Van Nostrand Company

[26] Kundu, D. and Howlader, H. (2010). Bayesian inference and prediction of the inverse Weibull distribution for type-II censored data. Computational Statistics and Analysis, 54: 15471558.

[27] Mahmoud, M. A. W., Sultan, K. S. and Amer, S. M. (2003). Order statistics from inverse Weibull distribution and associated inference. Computational Statistics \& Data Analysis, 42: $149-163$.

[28] Moors, J. J. A. (1988). A quantile alternative for kurtosis, Journal of the Royal Statistical Society. Series D (The Statistician), 37 (1): 25-32.

[29] Murthy, D. N. P., Xie, M. and Jiang, R.(2004). Weibull Models, John Wiley and Sons, New Jersey.

[30] Nelson, W. B. (1982). Applied Life Data Analysis. Wiley, New York. 
[31] R'enyi, A. (1961). On measures of entropy and information. In: Proceedings of the Fourth Berkeley Symposium on Mathematical Statistics and Probability-I, University of California Press, Berkeley, pp. 547-561.

[32] Sultan, K. S. (2008). Bayesian estimates based on record values from the inverse Weibull lifetime model, Quality Technology \& Quantitative Management, 5: 363-374.

[33] Zimmer, W. J., Keats, J. B., and Wang, F. K. (1998). The Burr XII distribution in reliability analysis. Journal of Quality Technology, 30: 386 - 394.

[34] Zografos, K., and Balakrishnan, N. (2009). On families of beta-and generalized gammagenerated distributions and associated inference. Statistical Methodology, 6: 344-362. 